\title{
De tablas rasas a sujetos encarnados
}

Olga Grau Duhart(1)

Resumen: El trabajo se propone abordar, de manera general, algunas de las metáforas relativas a la infancia que han sido parte de los modos en que la sociedad moderna se ha representado a las niñas y los niños (tablas rasas, ceras blandas, esponjas). Estas representaciones presentes en el imaginario cultural dominante determinaron, de manera significativa, no sólo los modos de relación concreta entre adultos y niños, sino también expectativas, condiciones y normativas del sistema educativo. Asimismo, se alude en el artículo a la concepción de infancia que está a la base del Programa de Filosofía para Niños y a una de sus categorías fundamentales, la comunidad de indagación, que producen una suerte de inversión en las valoraciones habituales respecto de los modos de entender la construcción del conocimiento y el régimen de influencias e interacciones entre adultos y niños.

En la época moderna fueron definidas, desde el pensamiento filosófico, un conjunto de categorías que articularon por varios siglos nuestras concepciones de mundo. Una determinación importante dentro de esas concepciones fue el modo de pensar la infancia, que podríamos relacionar con una de las elaboraciones teóricas que emergieron en la modernidad respecto del proceso de adquisición del conocimiento.

Recordemos que para Locke nuestras mentes son en primera instancia tablas rasas donde se graban las impresiones sensibles, materia prima de la construcción del conocimiento. Para los efectos de mi reflexión, y siguiendo con las posibilidades que nos da esta metáfora, la de la tabula rasa, me interesa pensar las ideas de marca, de huella, de impronta, de inscripción, de inculcación, que se le pueden acercar. Se graba en la tabla rasa (pizarra o página en blanco) lo que del afuera se imprime en esa superficie a través de la punta de tinta, cera o grafito, en el caso del papel, o del cincel y la gubia, en la tabla sin relieve, rasa, lisa, sin figuras propias.

Se podría aventurar, quizás, que la metáfora de la tabla rasa alude a una concepción de los procesos del conocimiento y del aprendizaje como escritura, constituyéndose, por tanto, en el núcleo significante para entender dichos procesos, que haría posible la separación entre naturaleza y cultura. La escritura llega a ser, desde esta perspectiva, como el conjunto de las marcas que separan, que nos separan de la naturaleza, aunque puedan provenir también de ésta. Lo que le ocurre a la mente, al entendimiento humano, de acuerdo a esta concepción, es recibir los signos de un mundo, fenómeno que se da igualmente en todos y a través del cual advenimos humanos. Esta imagen nos sugiere que la superficie lisa de la mente, como espacio virginal original e idéntico en los individuos, irá recibiendo lo 
externo a través de inscripciones que se superpondrán y mezclarán con otras, grabadas con anterioridad.

Pensemos ahora en uno de los conceptos más recurrentes que se han utilizado para la formación del carácter moral. Se ha hablado muchísimas veces y se sigue hablando, por parte de madres y padres y educadores, de la inculcación de valores. Nuevamente estamos frente a una palabra que alude a procesos de inscripción, que tendrá relación con el grabarse o imprimirse algo en una superficie, que nos puede llevar a imaginar el tatuaje, el maquillaje ritual, la inscripción de un nombre en un listado, y también la inscripción de los códigos culturales en nuestros comportamientos.

Las metáforas materializan nuestras concepciones y hacen visibles nuestras representaciones de mundo, $y$, en ese sentido es que podemos darles un lugar en nuestro análisis(2). Considerando la metáfora de la tabla rasa y la del concepto de inculcación, "repetición con ahínco"(3), que me sugieren a su vez el de inscripción, tal vez sería posible, relacionar la inscripción, como fenómeno cultural, con el poder. Y la idea de la inculcación me parece que lo permite. El concepto de inculcar contiene la idea de la repetición y la de la voluntad que hace que una idea se in-corpore haciéndose propia en otro, o que un deseo propio sea también de otro. La inculcación, como fenómeno de interacción social, podemos considerarla inserta en las retóricas del poder como persuasión o en su forma física, como golpe.

Para Locke, en sus reflexiones sobre la educación, los educadores no debían usar la violencia, y debían recurrir más bien al juego para activar los procesos de adquisición del conocimiento. Podemos pensar que en el siglo XVII el recurso a la violencia estaba muy presente y extendido en los espacios de enseñanza. Sin embargo, nosotros mismos podemos recordar algún hecho donde fuimos objetos de violencia por parte de nuestros profesores o profesoras, violencia que no podría sino concebirse como el deseo de dejar una marca indeleble que impone un sujeto a otro.

Recuerdo una escena, que forma parte de un episodio de mi propia infancia, en que una compañera del primer grado, saltarina, desordenada y de pelo colorín que lo llevaba en una melena con chasquilla, gustaba de trepar un árbol y encaramarse por donde fuera posible. En una ocasión, después de un recreo en que nuevamente había que formarse para entrar a la sala, procedimiento que forma parte de los disciplinamientos de los cuerpos, la chica había hecho caso omiso de la campana y del hecho de que nosostras ya nos habíamos formado. La profesora la hizo venir, la colocó al lado mío, tomó mi cabeza y la chocó violentamente contra de la de mi compañera que sujetaba con la otra mano. Quería inculcarle, y de paso también a mí y a todas las que estábamos en la fila, el sentido de la obediencia, del cumplimiento de las normativas, del reconocimiento de la autoridad, de los ordenamientos del tiempo y del espacio, de los mandatos de género. Deseo de marcar indeleblemente. 
Podríamos decir que ha pasado el tiempo entre mi generación y las nuevas. Pero, desgraciadamente, también hemos sabido de los recurrentes y actuales usos de la violencia no sólo en los hogares de menores, sino también en las salas cunas, los jardines infantiles, las escuelas. Violencia que va desde desde el abuso y violencia sexual, o desde el hacer comer el propio vómito cuando una niña o un niño no pueden con un alimento o con el ritmo con que se les hace comer, hasta los sofisticados sarcasmos debilitadores de la dignidad de niños y niñas o adolescentes.

No podría dejar de ver en el proyecto educativo de Summerhill, la conversión experimentada por Neil, su autor, sino a partir de la experiencia de golpear a un niño con su cinturón de cuero. El hecho lo golpeó a él mismo y decidió construir un nuevo modelo educativo en las antípodas de la educación castigadora. El cinturón de cuero, en la mano del adulto, es, en ese suceso, lo que marca, lo que vehiculiza el deseo de la inculcación de un valor o una norma en alguien considerado menor, de menor poder, de menor conocimiento, de menor desarrollo, de menor tiempo vivido. Con el golpe se ha querido imponer, domesticar, docilizar por siglos, y separar lo que se concibe amenazante para los ordenamientos dominantes.

La representación de la mente como tabla rasa, puede haber condicionado un tipo de relación con la niñez, concebida como receptáculo de impresiones, como superficie de impresiones, en la que no se ve o reconoce una configuración particular de procesos complejos y en gran medida imprevisibles, que exceden el significante de la impronta. En este punto quisiera señalar, como lo apunté en un texto escrito anteriormente, que la pregunta que hace la niña o el niño pequeños contiene ya un saber, una elaboración. La pregunta no es ausencia total del contenido por el que se pregunta, sino que ella incorpora observaciones, relaciones, de tal modo que se podría decir que algo más quieren saber de eso por lo que preguntan.

Por su parte, Lipman, el autor e impulsor de Filosofía para Niños, establecerá que los niños en la comunidad de indagación, entendida como escenario de aprendizajes que potencia el pensamiento autónomo, pueden, de algún modo imprevisiblemente, dar cuenta de habilidades que no son restricitivas de una fase determinada del desarrollo. Desde esta perspectiva, los niños y niñas pueden pensar filosóficamente desde muy pequeños, en cuanto piensan reflexivamente y con niveles de profundidad inesperados desde los cánones habituales de lo que constituye la comprensión de la niñez y la filosofía.

A mi juicio, la concepción de la infancia que está implicada en el Programa de Filosofía para Niños abre un horizonte que hace posible pensar una inversión en los valores y modos habituales de entenderse las relaciones intergeneracionales entre niños y adultos posibilitando dinámicas democráticas y comunitarias.

En la medida que en la comunidad de indagación, concepto clave en la propuesta de Lipman, se desdibujan los roles tradicionales, la horizontalidad propia de esta comunidad hace que el profesor aprenda de los saberes que poseen los niños $y$ niñas, de sus particulares percepciones y modos de pensar y reflexionar, de sus 
imaginarios, de su sensibilidad. De este modo, profesoras y profesores pueden jugar un rol que trasciende la transmisión y la regulación y puedan constituirse, más bien, en dadores de oportunidades y generadores de climas afectivos y sociocognitivos, a partir de los cuales tanto los niños y niñas como ellos mismos puedan hacer aprendizajes desde una experiencia común.

Pensamos que el modo de concebir la infancia en Filosofía para Niños, puede aportarnos una dimensión de extraordinario valor para el ejercicio de una práctica de creación de vínculos desjerarquizados y de reconocimiento y apreciación de las diferencias, que permita la reconstitución del sentido de comunidad, y de relaciones intergeneracionales de reciprocidad que enriquezcan los espacios educativos. Hannah Arendt señalaba la crisis de la educación y la interpretaba como crisis de relación entre generaciones y la necesidad de la reposición del amor por los jóvenes y los niños. En su interés filosófico y político, Hannah Arendt en su obra La Condición Humana, ofrece elementos para pensar la relación con los otros, entre otros, en virtud de la constitución de una comunidad posible.

A nuestro juicio, las concepciones de la infancia -conformadas por sistemas de representaciones sociales respecto de las identidades de niñas y niños y que configuran un sistema de dispositivos que estructuran las relaciones con el mundo adulto- han estado, fundamentalmente, influidas por un concepto reductivo e la infancia en varios sentidos.

Uno de estos sentidos se vincula con formas de socialización de carácter jerárquico, que impide relaciones intergeneracionales desde la reciprocidad o la horizontalidad y que posibilita prácticas de abuso de diverso orden hacia niños y niñas (que van desde el maltrato a la violencia sexual). Otro de los sentidos es la falta de espacios (entendidos tanto como espacios físicos como de climas educativos) que den efectivamente la oportunidad para la participación activa, donde puedan ser protagonistas en tanto sujetos generadores de cultura, ensayando procesos propios de culturización. Otro sentido reductor es la concepción de género de carácter discriminatorio, presente en el modo de concebir a niñas y niños, limitando las posibilidades amplias en la construcción de las subjetividades.

Si observamos de una manera extensa las prácticas educativas, familiares y escolares, de nuestra sociedad, podemos apreciar que en el imaginario social aún subsiste una concepción de infancia que tiene efectos limitantes en varios aspectos de nuestra cultura. Uno de esos efectos es el hecho de que niñas y niños no son validados y legitimados suficientemente como sujetos con grados de autonomía, como sujetos productores de cultura y como protagonistas de sus propios procesos de aprendizaje múltiple.

Hemos podido comprobar estas limitantes, a través de las experiencias realizadas en el Programa de Filosofía para Niños, experiencias de capacitación de docentes y de observación de clases. Pero también hemos podido observar, en el contacto con los niños y niñas en los talleres de Filosofía para Niños, que si se les da el espacio para la producción y circulación de sus reflexiones, indagaciones y creaciones, niños y niñas pueden, efectivamente, realizar un gran aporte en términos de ofrecer 
al mundo adulto otros modos de mirar la realidad y otras pasiones de vida. Ello requiere cambiar nuestros enfoques y educar la sensibilidad para acceder a los imaginarios infantiles de múltiple factura.

En una clase reciente, en el curso de Imaginario infantil que imparto junto a otras colegas en la Universidad de Chile, llevé una hermosa caracola para trabajar con ella. Me propuse dos objetivos. El primero, propiciar en las y los estudiantes el darse cuenta de las perspectivas en que siempre estamos situados. Quienes estaban sentados frente a mí, pudieron ver en un primer momento su superficie externa, llena de salientes, y cuyos terminaciones eran muy sugerentes. Dejé hacia mí su lado más acaracolado, para mostrarlo y sorprenderles posteriormente en su belleza, con su abertura de colores y matices, y su fondo que se pierde a la vista. Fue parte de la experiencia el reconocimiento de los puntos de vista, los que podían existir entre ellos y los que podían existir entre ellos y yo misma. Reconocer que siempre nuestro decir es desde un lugar y está atravesado por experiencias particulares, forma parte de la indispensable conciencia de nuestras posibilidades y de nuestras limitaciones. Estamos en el lugar que implica que no estamos en el lugar de los otros, y que, por tanto, nuestra experiencia es siempre limitada. Trascenderla es ir más allá de nuestros propios límites a través de la experiencia de los demás.

El segundo propósito con la caracola, era invitar a quienes estaban frente a mí, a través de la caracola, a viajar hacia su propia infancia; que el lado acaracolado les llevara a adentrarse en el mundo de los recuerdos, como modo de recuperar escenas tal vez olvidadas y que la caracola, al igual que el rumor del mar, podría traerles a la mente. Esta experiencia la entendíamos como experiencia propiciatoria de un proceso que puede llevar a empatizar con los modos de ser de niñas y niños, a reconocerles en su multiplicidad, en su diversidad.

Desde hace un tiempo me punza la idea de que somos lo que no somos. Somos aquello que nunca seremos, porque nuestra existencia se ha dado en un contexto específico; somos lo que no hemos podido ser (por circunstancias culturales, socioeconómicas, históricas, geográficas) o no nos hemos imaginado ser, o no hemos ensayado ser, y con el trayecto que nos ha sido posible, deseado, o inevitable. Somos un recorte en la realidad, un punto vivo en conexión con otros puntos vivos. Nuestra manera de trascender es a través de las experiencias y perspectivas que nos puedan ofrecer los demás, también a través de nuestra imaginación que se abre para acceder a esos otros mundos subjetivos. El ejercicio de ponerse en otro lugar, de situarnos en un lugar que no es el nuestro, que incluso nos puede ser muy infamiliar, es una condición para la comprensión de algo que está más allá de nosotros mismos.

A nuestro juicio, existe una tendencia homogeneizadora de concebir la infancia, pese a todos los intentos para que ocurra lo contrario, que pone en el olvido, pese a que se expliciten desde los discursos las particularidades de los contextos socioculturales locales, de la diversidad étnica y socioeconómica, de los condicionamientos culturales de género tan presentes en los procesos de socialización y culturización de niños y niñas (4). 
La comunidad de indagación que se practica en Filosofía para Niños, requiere del ejercicio de ver más allá de sí mismo y lo instala permanentemente como práctica de vida en comunidad, en la comprensión de los sujetos como sujetos encarnados, sexuados, generizados, insertos en contextos particularísimos que implican tanto la alimentación, el clima, como las sintaxis de la lengua en que se habla. Sujetos encarnados y cruzados por las representaciones que procesan y tramitan en sus propio imaginarios, que hacen suyos los diversos mandatos culturales $\mathrm{u}$ operan respecto de ellos una distancia crítica. Sujetos que han dejado deconcebirse a sí mismos como recipientes o superficies.

\section{Referencias a autores:}

ARENDT, Hannah. La Condición Humana. Barcelona: Edit. Paidós, 1993

LIPMAN, Matthew; SHARP, Ann M. Filosofía en el Aula. España: Editorial De la Torre, 1992.

LOCKE, John. Ensayo sobre el entendimiento humano.

NEILL, Alexander S. El nuevo Summerhill. México: Edit. Fondo de Cultura Económica, 1995.

VYGOTSKY, L. Pensamiento y lenguaje. Buenos Aires: Edit. Lautaro, 1964.

\section{Notas}

(1) Profesora de Filosofía en el Departamento de Filosofía y en el Centro de Estudios de Género y Cultura en América Latina, Universidad de Chile. Profesora en la Carrera de Educación Parvularia y Básica Inicial, Facultad de Ciencias Sociales, Universidad de Chile. Correo electrónico: ograu_2000@yahoo.com.

(2) En este punto debemos recordar también la recurrente metáfora de la esponja, (recepción pasiva, directa, sin mediación), referida a la condición de absorción por parte de los niños y niñas de lo que los adultos les transmiten, pudiendo reconocerse en estas metáforas representaciones habituales de los adultos respecto de ésos.

(3) Diccionario Ideológico de la Lengua Española, Editorial Bibliograf, Barcelona 1995.

(4) Vygotsky pondrá el acento en los contextos sociales y culturales en que se desarrollan los niños. El ambiente y el niño se encuentran en cambios constantes produciéndose influencia mutua, no siendo por tanto posible la determinación de patrones universales para el desarrollo. Vygotsky, plantea en su teoría sociocultural del lenguaje y del desarrollo cognoscitivo que el conocimiento no se construye de modo individual sino que los niños provistos de funciones elementales (percepción, 
memoria, atención y lenguaje) las transformarán en funciones mentales superiores a través de la interacción. Desde esta posición, que puede ser resumida en la categoría de Vigotsky "zona proximal de desarrollo", es posible pensar vínculos de reciprocidad en el cambio y aprendizajes significativos mutuos. 